\title{
Differential Effects of Haloperidol, Risperidone, and Clozapine Exposure on Cholinergic Markers and Spatial Learning Performance in Rats
}

\author{
Alvin V Terry $\mathrm{Jr}^{1,5}$, William D Hill ${ }^{2,5}$, Vinay Parikh ${ }^{3}$, Jennifer L Waller ${ }^{4}$, Denise R Evans ${ }^{3,5,6}$ and Sahebarao \\ P Mahadik*,3,5 \\ 'Program in Clinical and Experimental Therapeutics, College of Pharmacy (Augusta Campus), University of Georgia, USA; ${ }^{2}$ Department of Cell \\ Biology and Anatomy, Veterans Affairs Medical Center, Augusta, GA, USA; ${ }^{3}$ Department of Psychiatry and Health Behavior, Veterans Affairs \\ Medical Center, Augusta, GA, USA; ${ }^{4}$ Office of Biostatistics and Bioinformatics, Veterans Affairs Medical Center, Augusta, GA, USA; ${ }^{5}$ Medical \\ College of Georgia and Medical Research, Veterans Affairs Medical Center, Augusta, GA, USA; ${ }^{6}$ Mental Health Service Lines, Veterans Affairs \\ Medical Center, Augusta, GA, USA
}

Haloperidol (HAL), a potent typical antipsychotic, continues to be a frequently prescribed medication for behavioral disturbances associated particularly with schizophrenia despite well-documented adverse effects associated with its chronic use. Animal experiments have even indicated that HAL can damage cholinergic pathways and thus could be especially deleterious to those experiencing cognitive deficits. However, several recent clinical studies indicate that atypical antipsychotics may actually improve cognitive function in some patients, although this assertion requires further investigation. The purpose of this study was to compare the effects of prior chronic (45or 90-day) oral exposure to HAL and the atypical antipsychotics risperidone (RISP) and clozapine (CLOZ) on cognitive performance and central cholinergic markers in rats. All analyses were done after 4 days of drug washout in order to minimize direct drug effects. Learning performance and choline acetyltransferase (ChAT) levels were assessed in a water maze task and with immunofluorescence staining, respectively. HAL significantly impaired learning performance after 90 but not after 45 days of treatment when compared to both vehicle controls and the atypical agents, while RISP slightly improved task performance. Both 45 and 90 days of previous HAL exposure reduced ChAT staining in several brain regions, including the cortex, caudate-putamen, and hippocampus. ChAT staining in the caudate-putamen and hippocampus was also decreased after 90 days of RISP exposure, raising the possibility of deleterious cognitive effects after exposure to this dosage for longer periods of time. The results suggest that antipsychotic drugs exert differential and temporally dependent effects on central cholinergic neurons and learning performance.

Neuropsychopharmacology (2003) 28, 300-309. doi: I 0. I 038/sj.npp. 1300039

Keywords: antipsychotics; cognitive performance; choline acetyltransferase; risperidone; rat

\section{INTRODUCTION}

While delusions, hallucinations, and thought disorders are widely recognized characteristics of schizophrenia, cognitive dysfunction and memory impairment are also consistent and reproducible findings in this patient population (Tollefson, 1996; Friedman et al, 1999). In fact, it is generally acknowledged that the cognitive symptoms are probably the most debilitating as well as the most difficult to treat. While attention deficits and memory dysfunction have been documented in drug-naïve schizophrenics, it is

* Correspondence: SP Mahadik, Medical Research Service (242) Veterans Affairs, Medical Center, I Freedom Way, Augusta, GA 30904, USA, Tel: 706 733-0I 88 ext. 2490, Fax: 706 823-3977 or 3949, E-mail: mahadik@psychnts4.mcg.edu

Received 9 January 2002; revised 5 July 2002; accepted 19 July 2002 Online publication: 25 July 2002 at http://www.acnp.org/citations/ Npp072502358 not always readily apparent whether cognitive dysfunction is innate in a given patient or a result of drug therapy, since most patients diagnosed with the disease have a long history of exposure to neuroleptics (Lussier and Stip, 2001). Furthermore, many of the clinical studies conducted to date to investigate this issue have been confounded by variables such as the heterogeneity of study groups, florid symptoms in near drug-free states (to establish baseline data), polypharmacy, and the use of anticholinergic drugs to control extrapyramidal symptoms (Sharma and Mockler, 1998). A few studies have been conducted to address this issue in animal models and, to date, many of the available neuroleptic agents (ie both typical and atypical) have been observed to exert negative effects on memory. However, in most cases, only the acute effects of these drugs were measured. For example, Skarsfeldt (1996) found that haloperidol (HAL) and several atypical neuroleptics (clozapine (CLOZ), olanzapine, and risperidone (RISP)) impaired 
place navigation in the Morris Water Maze when administered $30 \mathrm{~min}$ before testing in a 4-day training regimen. Didriksen (1995) observed disruption of a delayed nonmatch to position paradigm in acute, dose-effect studies of a number of neuroleptic compounds (including HAL, CLOZ, RISP). Therefore, further animal studies designed to investigate the effects of chronic exposure to these agents are needed.

Another concern has arisen recently over the proper use of neuroleptic agents in those with progressive dementia. Typical neuroleptics (in particular, thioridazine and HAL) are commonly used to control delusions, hallucinations, agitation, and other disruptive behavioral symptoms associated with Alzheimer's disease (AD) and have been established as superior to placebo (Schneider et al, 1990). While some of these behavioral manifestations may be partially ameliorated, only a minority of patients with dementia actually improves on these medications. In fact, approximately $20 \%$ of patients actually worsen (Schneider et al, 1990; Risse and Barnes, 1986). HAL continues to be a frequently prescribed medication for these patients (possibly because of its comparatively low cost) despite its potential for a wide variety of deleterious side effects. Some of these effects could be of special significance in the setting of a progressively degenerating brain. Although most of the focus to explain these effects is still on the differential affinity binding (Kapur et al, 1999; Wadenberg et al, 2001) and receptor mechanisms (Moore et al, 1993; Bymaster et al, 1996; Skarsfeldt, 1996) of several neurotransmitters, HAL may also interfere with mitochondrial electron transport and protein synthesis and potentially generate oxidative metabolites, which are structurally similar to the known neurotoxins, MPTP, and $\mathrm{MPP}^{+}$(Barrientos et al, 1998; Prince et al, 1997; Subramanyam et al, 1991). Furthermore, HAL has been observed to decrease ChAT enzyme activity in the striatum and hippocampus and decrease ChAT immunoreactivity as indicated by an apparent reduction in the intensity and number of stained neurons and their processes (Mahadik et al, 1988). This finding could be of particular significance in the light of the well-documented role of septo-hippocampal cholinergic pathways in cognitive function. Degeneration of these pathways is among the most salient and reproducible neurochemical finding in postmortem brains from AD patients (Bartus, 2000). While gross abnormalities in the cholinergic system have not been demonstrated in schizophrenic brains postmortem, significant correlations between reduced ChAT levels and impaired cognitive performance have been observed (Karson et al, 1993, 1996).

It should be noted that some recent clinical studies suggest that atypical neuroleptics may in fact improve memory function in schizophrenics (Kern et al, 1999; Cuesta et al, 2001). This finding could indicate that these agents, despite costs, would be clearly preferred over older (typical) neuroleptics. However, to date, no systematic and controlled animal studies have been conducted to investigate the potentially 'positive' or 'negative' effects of chronic exposure to typical and atypical agents in drug naïve subjects. The purpose of this study was, thus, to compare HAL to the atypical neuroleptics CLOZ and RISP in chronic studies in a water maze task for effects on spatial learning and memory and for effects on ChAT immunoreactivity to determine if the compounds had deleterious effects on the cholinergic system. Both memory tests and histochemical measurements were conducted after a drug washout such that only sustained (ie nondirect) effects of drug exposure were measured.

\section{MATERIALS AND METHODS}

\section{Animals}

Male albino Wistar rats (225-250g) were obtained from Harlan Sprague-Dawley, Inc. (Indianapolis, IN) and housed in a temperature-controlled room $\left(25^{\circ} \mathrm{C}\right)$ with a 12-h light/ dark cycle. Upon arrival, each animal was provided with tap water and food (Purina Rat Chow) ad libitum for 1 week. Thereafter tap water was replaced with the solutions described below. All procedures employed during this study were reviewed and approved by the Medical College of Georgia Committee on Animal Use for Research (CAURE) and the Veterans Affairs Medical Center Subcommittee on Animal Use, and were consistent with AAALAC guidelines.

\section{Chronic Drug Administration}

All drugs were prepared daily and administered in solutions that replaced drinking water. This method was preferred over multiple IM injections to maintain more constant drug levels and to reduce stress and neuromuscular damage, which has been associated with these agents. HAL was dissolved in a $0.1 \mathrm{M}$ solution of citric acid (in water) and diluted $(1: 100)$ to deliver the final daily dose of drug. RISP and CLOZ were each dissolved in a $0.1 \mathrm{~N}$ solution of acetic acid and subsequently diluted $(1: 100)$ to administer the final daily dose of drug. Both citric acid and acetic acid controls $(N=8$ each $)$ as well as tap water controls $(N=12)$ were included in the study to assure that unanticipated behavioral effects of the vehicles were not present. Rats ( $N=30$ per drug group) were exposed to a single dose of drug. After 45 days, 15 animals were then given a 4-day washout to minimize the acute effects of drugs. Next, animals were tested in behavioral studies (see below) and then killed approximately $18 \mathrm{~h}$ after the last water maze trial, and the brains stored for future histochemical analyses. The remaining 15 animals (from the original group of 30) were continued on the same drug and dose for an additional 45 days (ie for a total of 90 days of continuous drug exposure) and then given a 4-day washout. Representative animals from the 90-day treatment group were subsequently tested in the water maze procedure and then killed as described above. The HAL dose $(2 \mathrm{mg} / \mathrm{kg} /$ day) was chosen based on previous studies (Mahadik et al, 1988) to obtain plasma levels, which are relevant to standard doses in humans and based on the fact that HAL is metabolized over 3 times faster in rats than in humans. The RISP $(2.5 \mathrm{mg} / \mathrm{kg} /$ day $)$ and $\mathrm{CLOZ}(20 \mathrm{mg} / \mathrm{kg} /$ day $)$ doses were selected based on the published reports (Skarsfeldt, 1996; Didriksen, 1995) that have demonstrated significant (acute) behavioral effects in rodents. These doses are somewhat higher (on a $\mathrm{mg} / \mathrm{kg}$ basis) than those most commonly used clinically. 


\section{Spatial Learning - Water Maze Test}

Testing apparatus. Water maze testing was performed in a black circular pool (diameter: $180 \mathrm{~cm}$; height: $76 \mathrm{~cm}$ ) made of plastic (Bonar Plastics, Noonan, GA) as described previously (Terry, 2000). The pool was filled to a depth of $35 \mathrm{~cm}$ of water (maintained at $25.0 \pm 1.0^{\circ} \mathrm{C}$ ) that covered an invisible (black) $10-\mathrm{cm}^{2}$ platform. The platform was submerged approximately $1.0 \mathrm{~cm}$ below the surface of the water and placed in the center of the northeast quadrant. The pool was located in a large room with a number of extra-maze visual cues, including highly reflective geometric images (squares, triangles, circles, etc) hung on the wall, diffuse lighting, and black curtains used to visually isolate the test rat from the experimenter and awaiting rats. The swimming activity of each rat was monitored via a ccTV camera mounted overhead, which relayed information including latency to find the platform, total distance traveled, time and distance spent in each quadrant, etc to a video tracking system (Poly-Track, San Diego Instruments, San Diego, CA). Tracking was accomplished via a white rat on a black background.

Hidden platform test. Each rat was given 4 trials/day for 4 consecutive days to find the hidden platform. A trial was initiated by placing the rat in the water facing the pool wall in one of the four quadrants (designated NE, NW, SE, SW). The daily order of entry into individual quadrants was randomized such that all four quadrants were used once every day. For each trial, the rat was allowed to swim a maximum of $90 \mathrm{~s}$, in order to find the platform. When successful the rat was allowed a 30 -s rest period on the platform. If unsuccessful, within the allotted time period, the rat was given a score of $90 \mathrm{~s}$ and then physically placed on the platform and also allowed the 30 -s rest period. In either case the rat was immediately given the next trial after the rest period.

Probe trials. On day 5, two probe trials (transfer tests) were given in which the platform was removed from the pool to measure 'spatial bias' (Morris, 1984). This was accomplished by measuring the time and distance traveled in each of the four quadrants and the number of crossings over the previous platform location (ie $10 \mathrm{~cm}^{2}$ area).

\section{Cholinergic Activity by ChAT-immunohistochemical Methods}

In order to determine the effects of chronic antipsychotic exposure on central cholinergic neurons, we evaluated the number of choline acetyltransferase (ChAT) immunopositive cells (as well as levels of immunoreactivity) in specific brain regions by an indirect immunofluorescence method followed by epifluorescence microscopy on perfusion fixed coronal sections of identical thickness, $30 \mu \mathrm{m}$, at Interaural $9.48 \mathrm{~mm}$ and Bregma $0.48 \mathrm{~mm}$ (Paxinos and Watson, 1998). Cortical areas 1-5 (cingulate, primary motor, S1, somatosensory, and piriform cortices, respectively) and subcortical areas 6-8 (basal forebrain, basal ganglia, and hippocampus, respectively) from all treatment groups were analyzed. Thus, important cholinergic pathways in the brain including the basal forebrain-cortical pathway, septo-hippocampal pathway, and striatum were analyzed. The procedure used (ie the perfusion, fixation, and tissue processing conditions) was developed (Mahadik et al, 1988) based on several reports that used monoclonal antibody to ChAT for analysis of cholinergic neurons (Eckenstein and Thoenen, 1982; Houser et al, 1983; Borges and Iversen, 1986; Connaughton et al, 1986). Staining was carried out by the modified original immunofluorescence method (Sternberger, 1979).

Rats were anesthetized with ether following the completion of chronic exposure to neuroleptics (and behavioral studies) and perfused through the left ventricle, initially with $200 \mathrm{ml}$ of saline containing $2 \%$ glucose and $2 \%$ sucrose, $\mathrm{pH} 7.2$, for $20 \mathrm{~min}$ at $25^{\circ} \mathrm{C}$, and then with fixative: $4 \%$ freshly prepared paraformaldehyde in $0.1 \mathrm{M} \mathrm{Na}$ phosphate buffer, $\mathrm{pH} 7.2$, containing $15 \%(\mathrm{v} / \mathrm{v})$ picric acid. The brains were then immediately removed, sectioned coronally into blocks, postfixed in the above fixative for $60 \mathrm{~min}$, washed several times with phosphate buffer at $40^{\circ} \mathrm{C}$, and sunk in $30 \%$ sucrose in phosphate buffer for $18 \mathrm{~h}$ at $40^{\circ} \mathrm{C}$ and then paraffin fixed. The tissue blocks were frozen over dry ice, and coronal cryostat sections $(30 \mu \mathrm{m})$ were cut from the forebrain. Sections were subsequently floated onto slides, deparaffinized with xylene, hydrated with successive ethanol concentrations (95, 80, 70\% 2 min each), dried at room temperature for $2 \mathrm{~h}$, and washed for $30 \mathrm{~min}$ in Trisbuffered saline (0.1 M Tris, $0.15 \mathrm{M} \mathrm{NaCI}, \mathrm{pH} 7.4)$ to remove traces of fixative as well as to rehydrate the sections. The antigen was retrieved by heating sections in $0.01 \mathrm{M}$ sodium citrate three to four times, $5 \mathrm{~min}$ each. The sections were then washed for 2 min with PBS ( $\mathrm{pH} 7.4$ ) and incubated in $0.8 \%$ Triton X-100 in Tris-buffered saline for $15 \mathrm{~min}$, in $3 \%$ fetal calf serum (FCS) for $15 \mathrm{~min}$, and then with ChAT antibody $(2 \mathrm{mg} / \mathrm{ml}, 1: 25$ diluted) in Tris-buffered saline containing $3 \%$ FCS, $0.5 \%$ Triton X-100, and $0.1 \%$ Na-azide overnight at $4{ }^{\circ} \mathrm{C}$ in a humidified chamber. The sections were then washed $3 \times 10 \mathrm{~min}$ with PBS containing $3 \%$ FCS, biotinylated antibody $(50 \mu \mathrm{l} /$ section as per Vector Elite ABC kit) was applied, washed and then the application reagent ( $1: 50$ biotinyl tyramide, $50 \mu \mathrm{l} /$ section) was applied for $8 \mathrm{~min}$ at room temperature; it was incubated for $1 \mathrm{~h}$ with fluoresceine conjugated antibody $(1: 500)$ in PBS (containing 3\% FCS, $0.2 \%$ Triton X-100), washed with PBS, and mounted with coverslip sealed with nailpolish. Negative controls included the omission of primary antibody and the replacement of primary antibody with rat Ig.

Image analyses. Brain sections from the same coronal level (ie selected based on specific anatomical landmarks) from each treatment group were analyzed with a Zeiss Axioplan-2 fluorescent microscope equipped with a CCD camera, PC computer, and Zeiss KS-300 image analyses software. Three to four slides were obtained per rat from the middle of three equal-length coronal sections. Within each slide, eight different regions (described earlier) of the brain slice were examined for total fluorescence staining and the total number of fluorescence-stained elements. This was accomplished by importing the digital images into the Zeiss KS-300 image analyses software and counting the total number of stained elements (fluorescence counts) and measuring the total fluorescence intensity (ie via a relative, densitometric measurement of the collective intensity of all the spots). 


\section{Statistical Analyses}

Water maze. During maze acquisition, the data were collapsed across trials for each day and averaged to obtain a mean performance for each animal. A nested, repeated measures analysis of variance was used to examine differences between treatments within day and differences between days within treatment for three outcome measures: latency, distance, and swim speed. For each ANOVA model, fixed effects included day (1,2,3, and 4) and treatment (vehicle, CLOZ, HAL, and RISP). The two-factor interaction between treatment and day was also included in the model. A rat nested within the treatment by day interaction was included as a random effect. One-way analysis of variance was used to compare the group performance of probe trials. In a few cases, ranks were used rather than the actual data values because of the non-normality of the data.

ChAT immunohistochemistry. Nested analysis of variance models were performed for the following outcomes, total area fluoresced and total number of fluoresced counts for two different sets of analyses. The first analysis examined differences between acute and chronic time periods within treatment group and differences between treatment groups within acute and chronic time periods within each of eight regions of the brain. The treatment variable consisted of four levels (vehicle, CLOZ, HAL, and RISP). Within each treatment group there were four rats. Three to four slides were obtained per rat and consisted of a single slice of the brain. Within each slide, the eight different regions of the brain slice were examined for fluorescence. Fixed effects included treatment and the acute or chronic indicator and random effects included rat nested within treatment by acute/chronic indicator interaction. The analysis was also performed combining cortical regions 1-5.

For all analyses, an overall $\alpha$ level of 0.05 was used to test for statistical significance. A Bonferroni adjustment to the $\alpha$ level was used to determine post hoc differences. All analyses were performed using SAS 8.2.

\section{RESULTS}

\section{Water Maze Testing}

Hidden platform test. The latencies and swim distances required to locate a hidden platform in the water maze for days 1-4 (after 45 or 90 days of neuroleptic exposure) are illustrated in Figure 1. There were no significant drug effects on performance after 45 days of exposure. There were notable differences, however, after 90 days of exposure. Statistical comparisons of latencies across the three groups revealed the following results: treatment effect, $\mathrm{F}(3,53)=2.75, p=0.05$; day effect, $\mathrm{F}(3,9)=42.9, p<0.001$; treatment $\times$ day interaction, $F(159,227)=2.22, \quad p=0.02$. Similar results were evident when distances were analyzed. Thus, after the drug or vehicle exposure for 90 days followed by a 4-day washout period, the rats generally learned to locate the hidden platform with progressively shorter latencies (and swim distances) across the 4 days of training. However, post hoc comparisons indicated that the rats treated with HAL exhibited significantly higher mean latencies (and distances swum) than vehicle-, CLOZ-, or
RISP-treated animals. Further analyses revealed that the RISP-treated animals actually performed more efficiently than vehicle-treated animals $(p=0.01)$ on day 2 of testing. The CLOZ-treated animals showed a similar, nearly significant $(p=0.07)$ difference in training efficiency on day 2 .

Transfer test (probe trials). The effects of the antipsychotics on "spatial bias" were evident as the number of crossing of over the previous platform location (i.e., $10 \mathrm{~cm}^{2}$ area) as well as the percent of time spent, and distance swum in the previous platform quadrant (i.e., the quadrant where the platform had been located during hidden platform trials). All treatment groups (vehicle and drugtreated) preferred the previous target quadrant (as opposed to the other three quadrants) after both 45 and 90 days of previous exposure as indicated by a greater than chance (ie $25 \%)$ percentage of time spent and distance swum in this region of the pool $(P<0.05$, one-tailed $t$-tests). Significant differences between treatment groups were not observed after 45 days of previous drug exposure for any of the probe trial measurements (data not shown). After 90 days of previous drug exposure, however, the number of platform crossings was reduced in the HAL group $(p<0.05)$ compared to vehicle controls as well as RISP-treated animals. Furthermore, animals previously treated with RISP crossed the previous platform area more than the vehicle or HAL-treated animals. A trend toward superior performance in both the CLOZ and RISP groups (compared to control and HAL) was apparent after 90 days of drug exposure $(\mathrm{F}(3,53)=2.04, p=0.1)$ in comparisons of the percent of time spent and distance swum in the previous target quadrant.

Swim speeds. Swim speeds (ie the latency to find the platform/distance swum in seconds) were compared daily across the treatment groups and appear in Figure 3. Statistical comparisons revealed significant differences between the drug groups after 90 but not 45 days of neuroleptic exposure, treatment effect, $\mathrm{F}(3,53)=5.29$, $p<0.01$; day effect, $\mathrm{F}(3,9)=23.17, p<0.001$; without a significant group $\times$ day interaction, $\mathrm{F}(159,227)=1.01, p=$ 0.37 . Post hoc analyses indicated that the animals previously exposed to HAL swam significantly $(p<0.05)$ slower than the CLOZ- and RISP-treated animals and exhibited a trend toward slower speed $(p=0.08)$ when compared to vehicle. Interestingly, the RISP-treated animals even swam slightly (but significantly, $p<0.05$ ) faster than controls.

\section{ChAT Immunofluorescence}

The results of ChAT immunofluorescence experiments are presented in Table 1. Representative photomicrographs of cortex, hippocampus, and caudate-putamen after 45 and 90 days of neuroleptic treatment appear in Figures 4, 5, and 6 , respectively. In cortical regions (Figure 4, region 1, cingulate cortex) neuropiles (synaptic terminals) are evident in the vehicle control, CLOZ, and RISP examples after both 45 and 90 days of exposure, whereas they are essentially absent in the HAL slides. Thus both the number of stained neurons and the level of fluorescence (intensity) were significantly $(p<0.05)$ reduced. A similar decrease in stained neurons and level of fluorescence was observed in 
45 Day Chronic Study
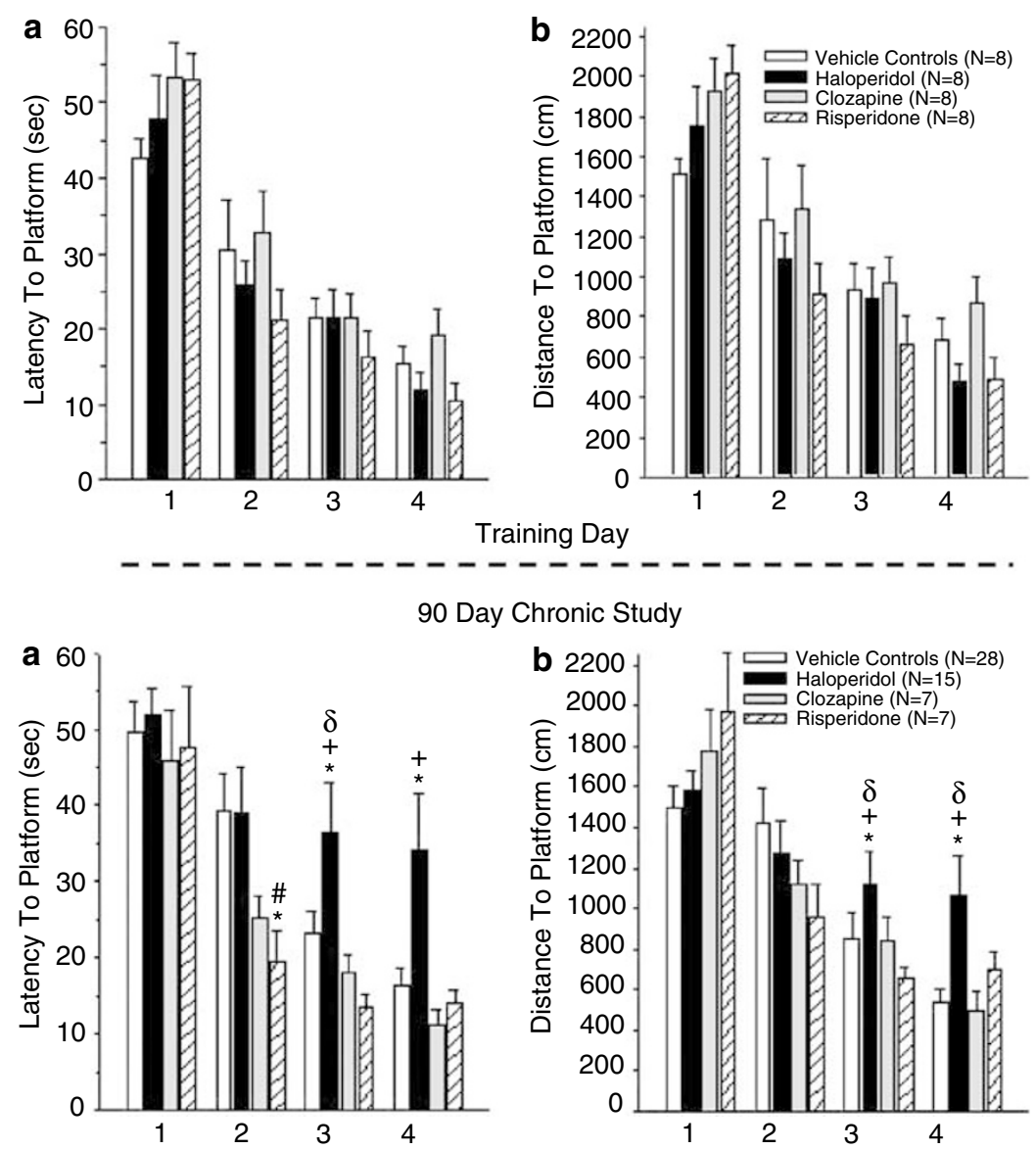

Training Day

Figure I Effects of chronic exposure to specific antipsychotic drugs on latency (a) and distances swum (b) to find a hidden platform in a water maze task on 4 consecutive days of testing compared to vehicle controls. Testing was conducted beginning after a 4-day washout from the various drugs. Top figure: 45 days of previous drug exposure. Bottom figure: 90 days of previous drug exposure. Each point represents the mean \pm SEM of 4 trials/day. *: significantly $(p<0.05)$ different from vehicle control value; \#: significantly different from HAL-associated performance; +: significantly different from CLOZ-associated performance; $\delta$ : significantly different from RISP-associated performance.

hippocampal slices (Figure 5, CA1 area). In addition, in the caudate-putamen region (Figure 6), both the number of stained neurons and the level of fluorescence were reduced after HAL exposure. Fluorescence (counts and intensity) was also reduced in the hippocampus and caudate-putamen region only after 90 days of RISP treatment, whereas up to 90 days of CLOZ treatment was not associated with reduced staining (Table 1).

\section{DISCUSSION}

The first notable finding of the present study was that 90 days of previous exposure by rats to HAL clearly resulted in impairment of spatial learning performance in the water maze task (as indicated by increased latencies and swim distances) when compared to vehicle controls. In contrast, the atypical agents CLOZ and RISP did not produce this effect and, in fact, RISP slightly improved the performance of the task on day 2 of testing. In this 4 trial/day paradigm, this is an unusual finding, since young animals generally perform at a level too high for significant improvement by cognitive enhancing agents to be observed (ie we generally use a 2 trial/day paradigm, age-impaired animals, or young animals impaired with cholinergic antagonists when testing potential cognitive enhancing agents). The (slightly) positive effects of RISP and the negative effects of HAL on memory-related task performance were further established in probe trials, platform area crossing experiments. The water maze test used (a version of the Morris Water maze) was selected for this study for several important reasons (Morris, 1984). First, accurate performance of the task requires intact cholinergic function, which we hypothesized (based on previous studies) might be damaged with prolonged exposure to neuroleptics. Second, the task employs the hippocampus and other medial temporal lobe structures known to be of importance to human cognition. Finally, the task requires many of the same processes known to be important components of human mnemonic function including information acquisition and processing, consolidation, retention, and retrieval (McNamara and Skelton, 1993; McDonald and White, 1995).

It should also be noted that longer swim distances associated with 90 days of HAL exposure (compared to 
vehicle controls and the other neuroleptics) were proportional to the longer latencies. This indicates that the negative effects of HAL in the water maze task were likely
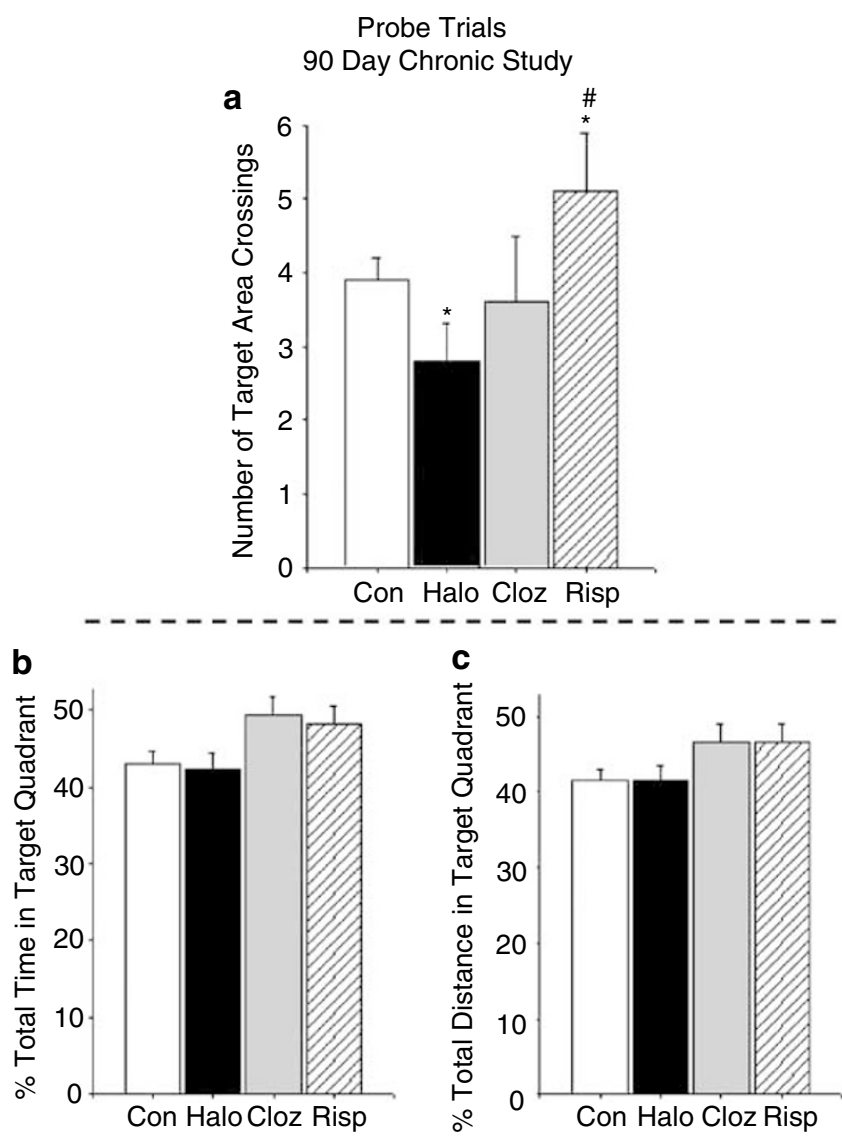

Figure 2 Effects of 90 days of previous exposure to specific antipsychotic drugs on water maze transfer tests (probe trials). Each bar represents the mean $( \pm$ SEM) of (a) number of crossings over the previous $10 \mathrm{~cm}^{2}$ platform area; (b) percentage of the total time spent in the previous target quadrant, or (c) percentage of total distance swum in the previous target quadrant $*$ : significantly different from vehicle control value; \#: significantly different from HAL value, one-way AVOVA $(p<0.05)$. to be memory-related. The distance measurements in this study are particularly important as are swim speeds (see below) because HAL has been observed to have adverse effects on motor systems (ie the striatum) in both animals and humans with chronic exposure. Through the use of the video tracking system (and software program), we were able to view (as well as record) the actual swim paths, turns, and headings associated with every trial for each test subject. This allowed us to examine search behaviors for potential nonmnemonic activities or strategies such as taxon, praxis, or thigmotaxis. We found none of these behaviors in any of the treatment groups (ie including the HAL-treated group). HAL was, however, associated with reduced swim speeds compared to all the other treatments, and thus some level of drug influence on motor activity cannot be ruled out. However, as indicated in the probe trials on day 5 , the animals administered HAL did prefer the previous target quadrant as opposed to the other quadrants of the pool. Probe trials generally measure the ability of the rats to

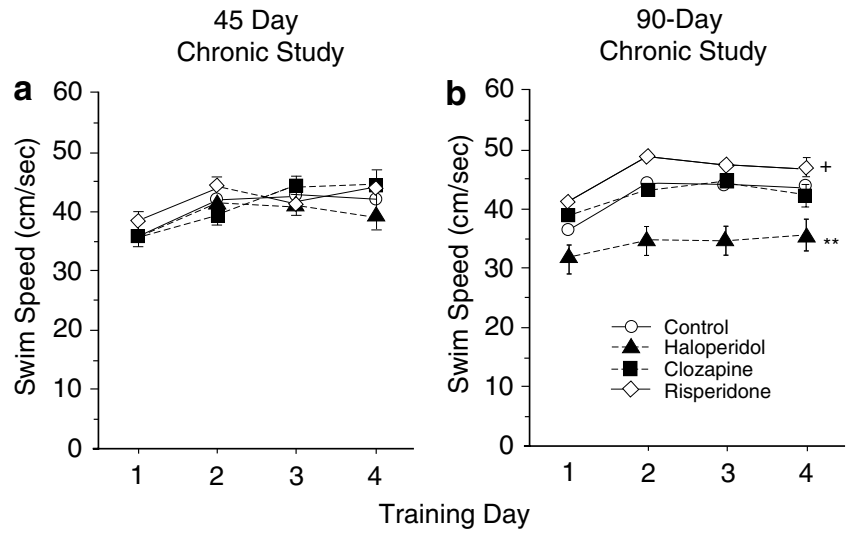

Figure 3 Effects of 45 days (a) or 90 days (b) of continuous exposure to specific antipsychotic drugs on swim speeds in a water maze task on 4 consecutive days of testing. Each bar represents the mean (cm traveled/ s) \pm SEM of 4 trials/day across the first 4 days of testing. +: significantly different from vehicle control value $(p<0.05)$; **: significantly different from CLOZ- and RISP-associated swim speeds.

Table I Choline Acetyltransferase Immunoreactivity

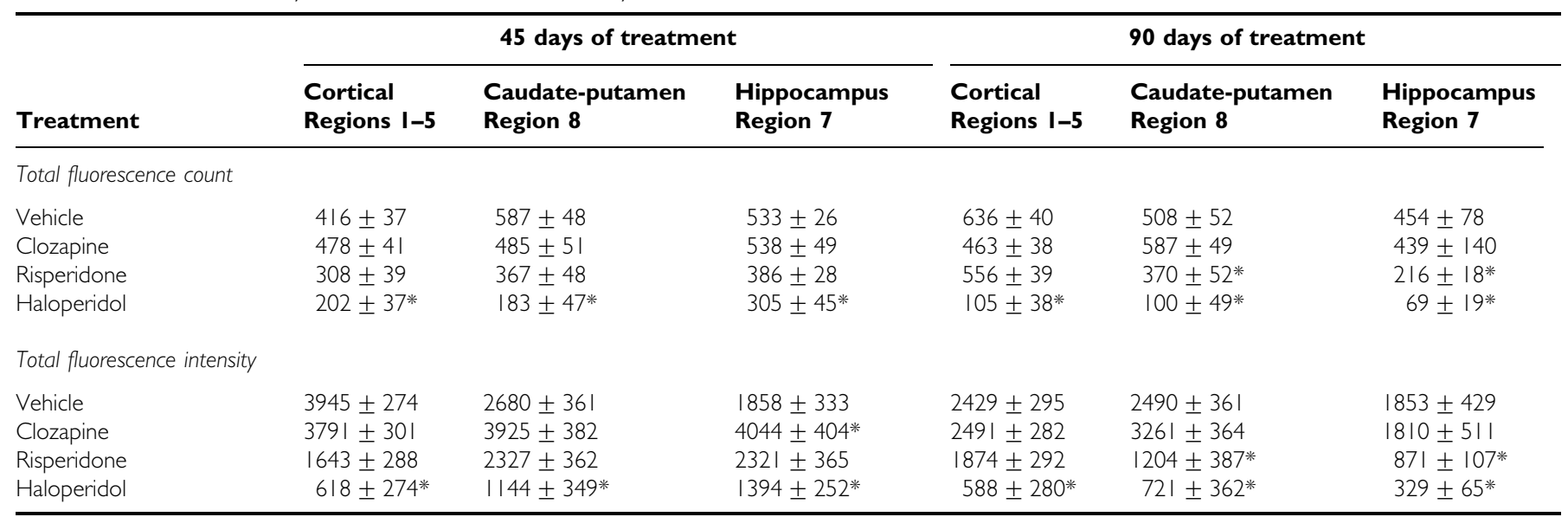

*Significantly different from vehicle controls $(p<0.05)$. 
45 day Exposure
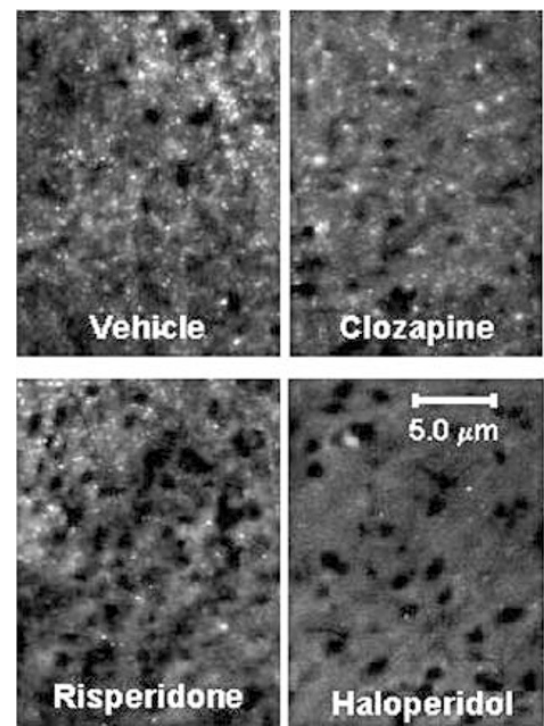

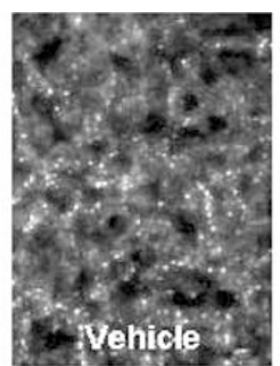

90 day Exposure
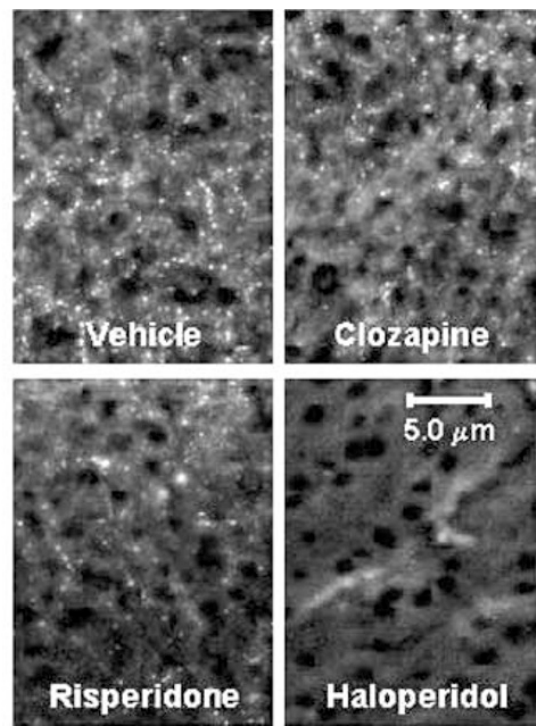

Figure 4 Representative photomicrographs illustrating the number of ChAT-stained elements and their intensity in the cortex (region I, cingulate cortex) after 45 or 90 days of continuous exposure to the antipsychotic agents. Stained neuropiles are evident after exposure to all of the compounds with the exception of HAL.

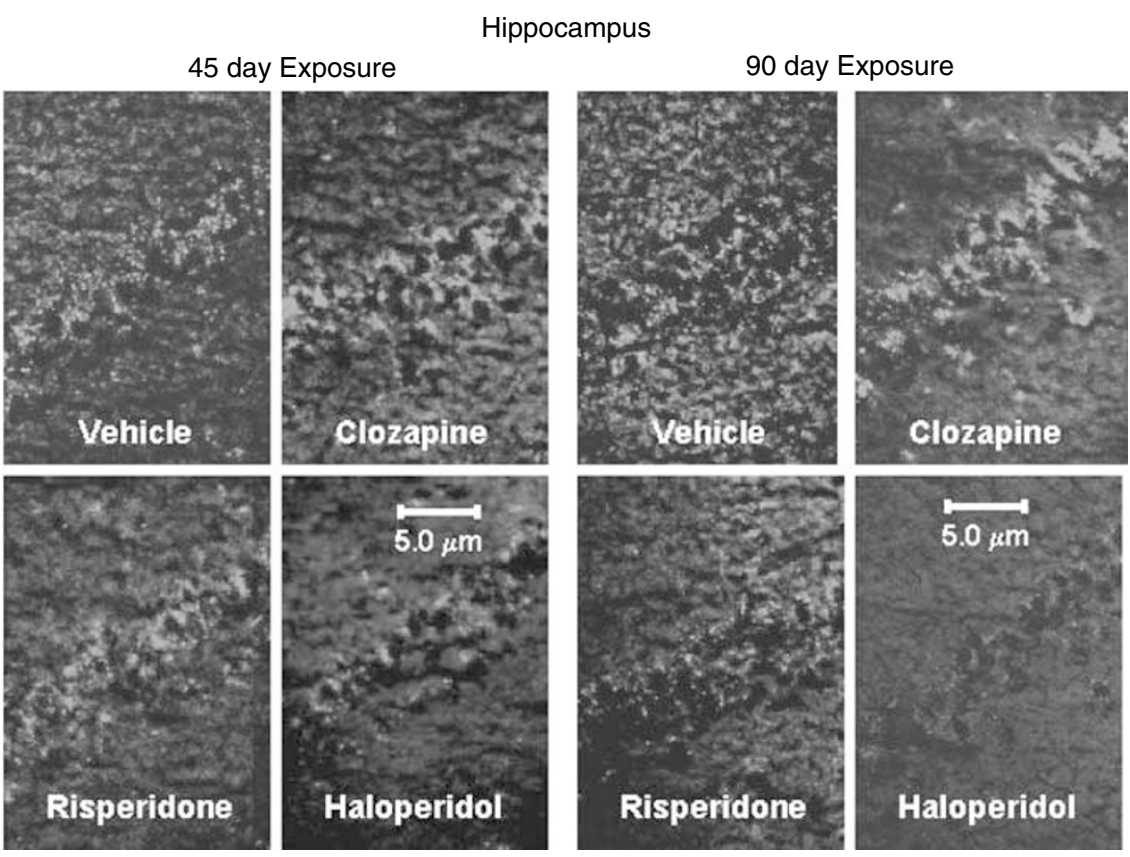

Figure 5 Representative photomicrographs illustrating the number of ChAT-stained elements and their intensity in the hippocampus (CAI area) after 45 or 90 days of continuous exposure to the antipsychotic agents. Stained neuropiles are evident after exposure to all of the drugs with the exception of HAL exposure for 90 days.

retrieve information learned during the first 4 days of testing (specifically, the strength and accuracy of this information). Since approximately $25 \%$ of the time spent and distance traveled in the previous target quadrant would be encountered if animals were randomly swimming about the pool (since there are four quadrants), it is obvious that significant learning in the HAL group did occur. Further- more, in the first 4 days of testing, a significant effect of the day of testing was evident for all the treatments. In the case of HAL, performances on days 2, 3, and 4 were all significantly different $(p<0.05)$ from that of day 1 , indicating improved learning of the task. Finally, in reference to the probe trial platform crossing data, it should be noted that the slightly enhanced swim speeds (in the case 


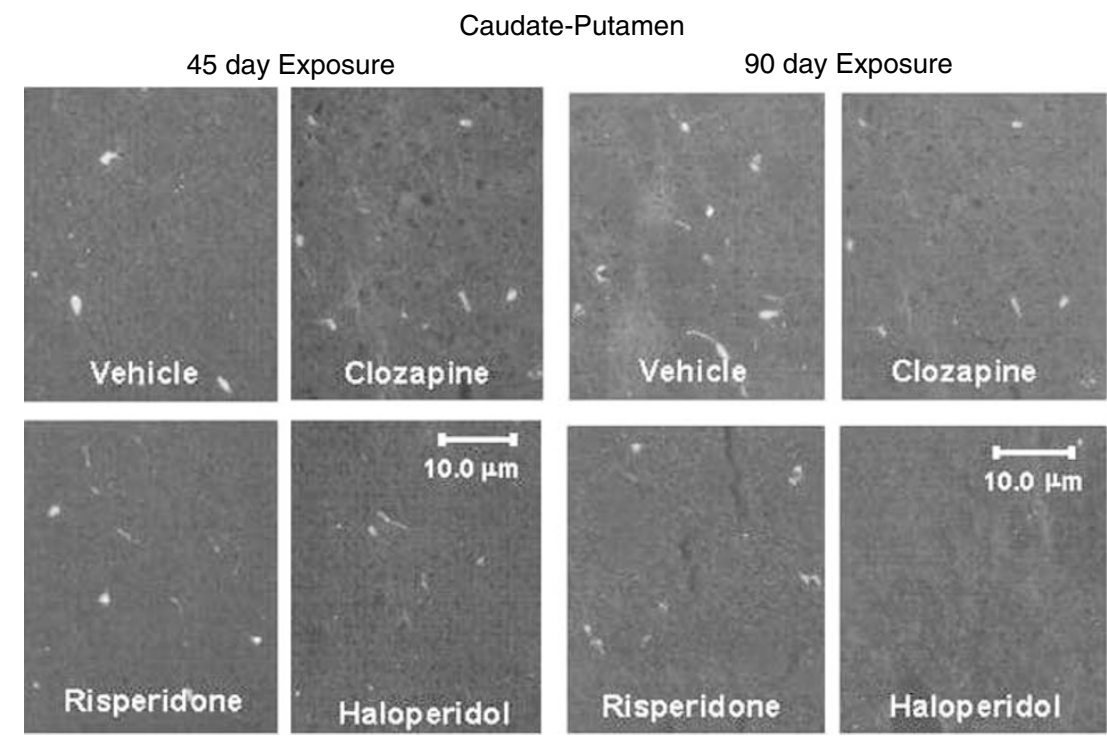

Figure 6 Representative photomicrographs illustrating the number of ChAT-stained elements and their intensity in the caudate-putamen region after 45 or 90 days of continuous exposure to the antipsychotic agents. Note the reduced level of staining in the HAL slides after both exposure periods.

of RISP) and the decreased swim speeds (in the HAL group, described above) could account for differences in platform crossings in probe trials. Observational analysis of search behaviors (head turns and slowing in the platform area) was more apparent in the RISP group (and diminished in the HAL group), however.

The immunohistochemical studies confirmed several earlier observations that HAL treatment negatively alters central cholinergic systems (Pedata et al, 1980; Mahadik et al, 1988; Korenovsky et al, 1990; Miller and Chouinard, 1993; Angelucci et al, 2000). These effects support the cognitive deficits observed after HAL exposure. Reduced ChAT staining was observed after 45 days of continuous exposure to HAL; however, even up to 90 days of CLOZ did not cause significant changes. RISP, in contrast, with the dosage used did reduce ChAT staining after 90 days of exposure, an observation that may indicate that a longer time of exposure could in fact result in cognitive deficits (since decreases in ChAT staining preceded the cognitive changes in the case of HAL). Collectively, the immunohistochemical and behavioral data collected in this study indicate that specific antipsychotic agents exert differential and temporally dependent effects on cholinergic markers and memory function. Considering the fact that these agents are commonly used for extended periods in human patients, it is essential that the dose-dependent chronic effects of all of the commonly used antipsychotic agents be further delineated.

The mechanisms associated with morphological and behavioral effects of antipsychotics may be very complex. However, these effects are unlikely to be related to direct drug actions, since analyses were done after 4 days of drug washout. It has been suggested that differential receptor occupancy may be one of the mechanisms (Kapur et al, 1999; Wadenberg et al, 2001). However, this may be inadequate to explain the persistent long-term effects, unless these receptor interactions trigger a series of biochemical processes that eventually lead to the effects we have observed. There is another mechanism that is also very compelling to explain a wide range of behavioral and pathological effects in schizophrenia after chronic HAL treatment, that is, free radical pathology. There is substantial evidence that HAL treatment increases free radical pathology in rat brain (Cadet and Lohr, 1987; Mahadik et al, 1999). In additional experiments (using some remaining tissues from the present study as well as additional animals treated with olanzapine or vehicle), we have detected changes in antioxidant enzymes and parallel free radicalmediated lipid peroxidation in the brain associated with HAL (Parikh et al, 2002). However, such changes were not found in animals treated with any of the atypical antipsychotics, RISP, CLOZ, or olanzapine. There is also substantial evidence for altered free radical metabolism and associated free radical pathology, lipid peroxidation (Mahadik and Mukherjee, 1996; Reddy and Yao, 1996; Tsai et al, 1998; Mahadik et al, 1999), and altered lipid metabolism (Peet et al, 1999) in schizophrenia. Some studies have also found that treatment with atypical antipsychotics, but not HAL, reduces the free radical pathology and improves membrane lipid integrity with parallel improvements in psychopathology (Horrobin, 1999; Khan et al, 2002; Arvindakshan et al, 2002).

The recent observations of Angelucci et al (2000) in which chronic HAL and RISP treatment decreased nerve growth factor (NGF) immunoreactivity in the striatum as well as the hippocampus also appear to be particularly interesting in the light of our results (ie reduced ChAT staining in each of these areas and memory dysfunction after chronic exposure to HAL). The observations are reminiscent of several studies in aged animals or subjects with experimental lesions, which have shown direct correlations between reduced NGF levels (or NGF receptor expression), decreased levels of the cholinergic enzyme, ChAT, and spatial learning deficits (Winkler et al, 2000). 
Collectively, the findings of this study indicate that the chronic administration of HAL leads to persistent neurochemical changes in the brain that appear to lead to cognitive dysfunction. This may indicate that HAL is a particularly undesirable neuroleptic choice for patients suffering from memory dysfunction, especially those in which cholinergic abnormalities may already be present (eg schizophrenia, AD, dementia with Lewy bodies, etc). In contrast, our results also indicate that the atypical agent CLOZ does not have this limitation. While RISP might have the potential to improve memory function, the fact that it was associated with reduced ChAT staining in the hippocampus and caudate-putamen after 90 days of exposure suggests that longer exposure periods may be deleterious to cognitive function.

In summary, while typical neuroleptics such as HAL are inexpensive and well documented to be of therapeutic benefit to patients with schizophrenia (and other conditions that present with psychotic manifestations), the wide array of known adverse effects (eg drug-induced parkinsonism, akathesia, dystonia, etc) as well as the findings of this study suggest that atypical compounds such as CLOZ are superior choices for long-term administration.

\section{ACKNOWLEDGEMENTS}

The authors would like to thank Joe Carothers, Kavya Sebastian, and Deepti Jain for their excellent technical assistance, and Dr Jeffrey Rausch for a critical review of the manuscript. This study was partially supported by Janssen Pharmaceutica Research Foundation, USA.

\section{REFERENCES}

Angelucci F, Aloe L, Gruber HM, Fiore M, Mathe AA (2000). Chronic antipsychotic treatment selectively alters nerve growth factor and neuropeptide $\mathrm{Y}$ immunoreactivity and the distribution of choline acetyltransferase in rat brain regions. Int $J$ Neuropsychopharmacol 3: 13-25.

Barrientos A, Marin C, Miro O, Casademont J, Gomez M, Nunes V et al (1998). Biochemical and molecular effects of chronic HAL administration on brain and muscle mitochondria of rats. $J$ Neurosci Res 53: 475-481.

Bartus RT (2000). On neurodegenerative diseases, models, and treatment strategies: lessons learned and lessons forgotten a generation following the cholinergic hypothesis. Exp Neurol 163: 495-529.

Borges LF, Iversen SD (1986). Topography of choline acetyltransferase immunoreactive neurons and fibers in the rat spinal cord. Brain Res 362: 140-148.

Bymaster FP, Hemrick-Luecke SK, Perry KW, Fuller RW (1996). Neurochemical evidence for antagonism by olanzapine of dopamine, serotonin, $\alpha 1$-adrenergic and muscarinic receptors in vivo in rats. Psychopharmacology 124: 87-94.

Cadet JL, Lohr JB (1987). Free radicals and the developmental pathology of schizophrenic burnout. Integrative Psychiatry 5: 40-48.

Connaughton M, Priestley JV, Sofroniew MV, Eckenstein F, Cuello AC (1986). Inputs to motoneurons in the hypoglossal nucleus of the rat: light and electron microscopic immunocytochemistry for choline acetyltransferase, substance $\mathrm{P}$ and enkephalins using monoclonal antibodies. Neuroscience 17: 205-224.
Cuesta MJ, Peralta V, Zarzuela A (2001). Effects of olanzapine and other antipsychotics on cognitive functions in chronic schizophrenia: a longitudinal study. Schizophr Res 48: 17-28.

Didriksen M (1995). Effects of antipsychotics on cognitive behavior in rats using the delayed non-match to position paradigm. Eur J Pharmacol 28: 241-250.

Eckenstein F, Thoenen H (1982). Production of specific antisera and monoclonal antibodies to choline acetyltransferase: characterization and use for identification of cholinergic neurons. EMBO J 1: 363-368.

Friedman JI, Harvey PD, Kemether E, Byne W, Davis KL (1999). Cognitive and functional changes with aging in schizophrenia. Biol Psychiatry 46: 921-928.

Horrobin DF (1999). The effects of antipsychotic drugs on membrane phospholipids - a possible novel mechanism of action of clozapine. In: Peet M, Glen I, Horrobin D (eds). Phospholipid Spectrum Disorders in Psychiatry. Marius Press: Lancashire, UK. pp 113-117.

Houser CR, Crawford GD, Barber RP, Salvaterra PM, Vaughn JE (1983). Organization and morphological characteristics of cholinergic neurons: an immunocytochemical study with a monoclonal antibody to choline acetyltransferase. Brain Res 266: 97-119.

Kapur S, Zipursky RB, Remington G (1999). Clinical and theoretical implications of 5-HT and D2 receptor occupancy of clozapine, risperidone and olanzapine in schizophrenia. Am J Psychiatry 156: 286-293.

Karson CN, Casanova MF, Kleinman JE, Griffin WS (1993). Choline acetyltransferase in schizophrenia. Am J Psychiatry 150: 454-459.

Karson CN, Mrak RE, Husain MM, Griffin WS (1996). Decreased mesopontine choline acetyltransferase levels in schizophrenia. Correlations with cognitive functions. Mol Chem Neuropathol 29: 181-191.

Kern RS, Green MF, Marshall Jr BD, Wirshing WC, Wirshing D, McGurk SR et al (1999). Risperidone versus HAL on secondary memory: can newer medications aid learning? Schizophr Bull 25: 223-232.

Khan MM, Evans DR Gunna V, Scheffer RE, Parikh VV, Mahadik SP (2002). Reduced erythrocyte membrane essential fatty acids and increased lipid peroxides in schizophrenia at the nevermedicated first-episode of psychosis and after years of treatment with antipsychotics. Schizophr Res 58: 1-10.

Kinon BJ, Lieberman JA (1996). Mechanisms of action of atypical antipsychotic drugs: a critical analysis. Psychopharmacology (Berl) 124: 2-34.

Korenovsky A, Laev H, Mukherjee S, Mahadik SP (1990). Quantitative analyses of plasma cholinesterase isozymes in HAL-treated rats. Biol Psychiatry 27: 871-883.

Lussier I, Stip S (2001). Memory and attention deficits in drug naïve patients with schizophrenia. Schizophr Res 48: 45-55.

Mahadik SP, Laev H, Korenovsky A, Karpiak SE (1988). HAL alters rat CNS cholinergic system: enzymatic and morphological analyses. Biol Psychiatry 24: 199-217.

Mahadik SP, Mukherjee S (1996). Free radical pathology and antioxidant defense in schizophrenia: a review. Schizophr Res 19: 1-18.

Mahadik SP, Sitasawad V, Mulchandani M (1999). Membrane peroxidation and the neuropathology of schizophrenia. In Peet M, Glen I, Horrobin D (eds). Phospholipid Spectrum Disorders in Psychiatry. Marius Press: Lancashire, UK. pp 99-111.

McDonald RJ, White NM (1995). Hippocampal and nonhippocampal contributions to place learning in rats. Behav Neurosci 109: $579-593$.

McNamara RK, Skelton RW (1993). The neuropharmacological and neurochemical basis of place learning in the Morris water maze. Brain Res Rev 18: 33-49.

Miller R, Chouinard G (1993). Loss of striatal cholinergic neurons as a basis for tardive and L-dopa-induced dyskinesias, neuroleptic-induced supersensitivity psychosis and refractory schizophrenia. Biol Psychiatry 34: 713-738. 
Moore NA, Calligaro DO, Wong DT, Bymaster F, Tye NC (1993). The pharmacology of olanzapine and other new antipsychotic agents. Curr Opin Invest 2: 299.

Morris RGM (1984). Development of a water-maze procedure for studying spatial learning in the rat. J Neurosci Methods 11: 4760.

Parikh V, Khan MM, White JR, Buckley PF, Mahadik SP (2002). Atypical antipsychotics such as risperidone and clozapine do not induce the oxidative stress and the lipid peroxidation similar to HAL in rats. Biol Psychiatry 51: 184S.

Paxinos G, Watson CR (1998). The Rat Atlas in Stereotaxic Coordinates, 4th edn. Academic Press: San Diego, CA.

Pedata F, Sorbi S, Pepeu G (1980). Choline high-affinity uptake and metabolism and choline acetyltransferase activity in the striatum of rats chronically treated with neuroleptics. J Neurochem 35: 606-611.

Peet M, Glen I, Horrobin D (1999). Phospholipid Spectrum Disorders in Psychiatry. Marius Press: Lancashire, UK.

Prince JA, Yassin MS, Oreland L (1997). Neuroleptic-induced mitochondrial enzyme alterations in the rat brain. J Pharmacol Exp Ther 280: 261-267.

Reddy R, Yao J (1996). Free radical pathology in schizophrenia: a review. Prostaglandins Leukot Essent Fatty Acids 55: 33-43.

Risse SC, Barnes RJ (1986). Pharmacologic treatment of agitation associated with dementia. J Am Geriatric Soc 34: 368-376.

Schneider LS, Pollock VE, Lyness SA (1990). A meta-analysis of controlled trials of neuroleptic treatment in dementia. $\mathrm{J} \mathrm{Am}$ Geriatric Soc 38: 553-563.
Sharma T, Mockler D (1998). The cognitive efficacy of atypical antipsychotics in schizophrenia. J Clin Psychopharmacol 18(2 Suppl 1): 12S-19S Related Articles, Books, Link Out.

Skarsfeldt T (1996). Differential effect of antipsychotics on place navigation of rats in the Morris water maze. A comparative study between novel and reference antipsychotics. Psychopharmacology (Berl) 124: 126-33.

Subramanyam B, Pond SM, Eyles DW, Whiteford HA, Fouda HG, Castagnoli Jr N (1991). Identification of potentially neurotoxic pyridinium metabolite in the urine of schizophrenic patients treated with HAL. Biochem Biophys Res Commun 181: 573-578.

Terry Jr AV (2000). Spatial navigation (water maze) tasks. In Buccafusco JJ (ed). Behavioral Methods in Neuroscience. CRC Press: Boca Raton, FL. pp 153-166.

Tollefson GD (1996). Cognitive function in schizophrenic patients. J Clin Psychiatry 57(Suppl 11): 31-39.

Tsai G, Goff DC, Chang RW, Flood J, Baer L, Coyle JT (1998). Markers of glutaminergic neurotransmission and oxidative stress associated with Tardive dyskinesia. Am J Psychiatry 155: 1207-1213.

Wadenberg M-LG, Soliman A, Vanden-Spek SC, Kapur S (2001). Dopamine D2 receptor occupancy is a common mechanism underlying animal models of antipsychotics and their clinical effects. Neuropsychopharmacology 25: 633-641.

Winkler J, Ramirez GA, Thal LJ, Waite JJ (2000). Nerve growth factor (NGF) augments cortical and hippocampal cholinergic functioning after p75NGF receptor-mediated deafferentiation but impairs inhibitory avoidance and induces fear-related behaviors. J Neurosci 20: 834-844. 